\title{
What Internal Variables Affect Sensorimotor Rhythm Brain-Computer Interface (SMR-BCI) Performance?
}

Alex J. Horowitz, DO, ${ }^{1,6}$ Christoph Guger, Dr.techn., ${ }^{2}$ Milena Korostenskaja, PhD ${ }^{1,3,4,5}$

\section{Abstract}

\section{Description}

In this review article, we aimed to create a summary of the effects of internal variables on the performance of sensorimotor rhythm-based brain computer interfaces (SMR-BCls). SMR-BCls can be potentially used for interfacing between the brain and devices, bypassing usual central nervous system output, such as muscle activity. The careful consideration of internal factors, affecting SMR-BCl performance, can maximize $\mathrm{BCl}$ application in both healthy and disabled people. Internal variables may be generalized as descriptors of the processes mainly dependent on the $\mathrm{BCl}$ user and/or originating within the user. The current review aimed to critically evaluate and summarize the currently accumulated body of knowledge regarding the effect of internal variables on SMR-BCl performance. The examples of such internal variables include motor imagery, hand coordination, attention, motivation, quality of life, mood and neurophysiological signals other than SMR. We will conclude our review with the discussion about the future developments regarding the research on the effects of internal variables on SMR-BCl performance. The end-goal of this review paper is to provide current $\mathrm{BCl}$ users and researchers with the reference guide that can help them optimize the SMR-BCl performance by accounting for possible influences of various internal factors.

\section{Keywords}

$\mathrm{BCl}$ adoption rates; amyotrophic lateral sclerosis (ALS); attention; brain-computer interfaces (BCls); BCl accuracy; BCl literacy; BCl performance; depression; distraction; electroencephalography (EEG); event-related desynchronization (ERD); information transfer rate (ITR); internal variables; mental state; motor imagery; mood; motivation; neuroprosthetics; quality of life (QoL); psychological variables; sensorimotor rhythm (SMR); signal classification accuracy

\section{Introduction}

A brain computer interface $(\mathrm{BCl})$ is a device that records and translates the user's brain activity into various command signals, thus bypassing muscle activity and allowing direct communication between the brain and various devices. Guger et al. defined BCls as "communication systems that allow people to send messages or commands without movement." Electromagnetic brain activity for $\mathrm{BCl}$ control can be recorded by a set of sensors when using magnetoencephalography (MEG), by a set of electrode arrays placed on the scalp when employing electroencephalography (EEG), as well as by electrode grids placed directly on the cortical surface when utilizing electrocorticography (ECoG). ${ }^{2}$ Figure 1 demonstrates these methods of recording electromagnetic brain activity. We limited the scope of this review article to the $\mathrm{BCls}$ driven by electrical signals that are recorded non-invasively, as this is one of the $\mathrm{BCl}$ types that is currently the most suitable for application outside the controlled laboratory settings. The recorded brain activity is further processed by the $\mathrm{BCl}$ according to a pre-defined fixed or changing ("adaptive") algorithm that translates the acquired signal in real time into the computer commands. This allows control of the devices that might be placed both within or outside of the $\mathrm{BCl}$ user. Figure 2

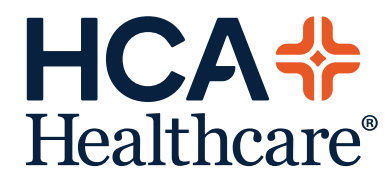

www.hcahealthcarejournal.com

(C) 2021 HCA Physician Services, Inc. d/b/a Emerald Medical Education
HCA Healthcare Journal of Medicine 


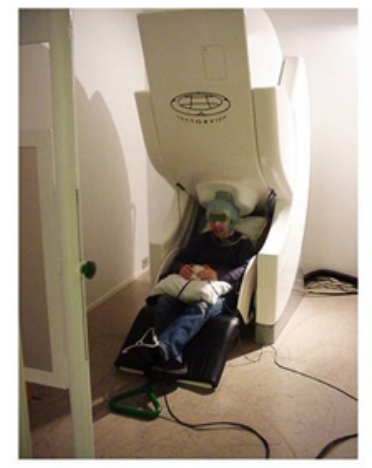

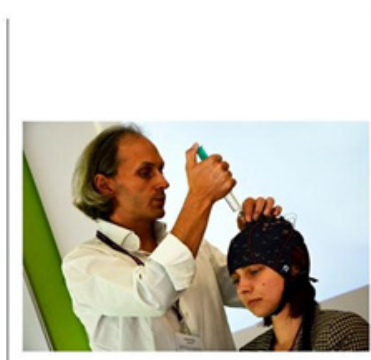

EEG

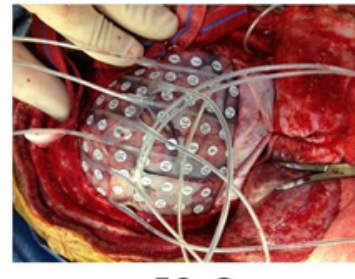

ECoG

MEG

Figure 1. Recording of magnetic (MEG) and electric (EEG, ECOG) brain activity that can be used for Brain-Computer Interface (BCl) applications. Left: Example of magnetoencephalography (MEG) at BioMag Laboratory, Helsinki University Central Hospital; Middle: Example of electroencephalography (EEG) at the Department of Biophysics, Vilnius University; Right: Example of electrocorticography (ECOG) at the Comprehensive Epilepsy Surgery Center, AdventHealth Orlando. (Photographs courtesy of the authors.)

depicts this closed-loop system for the operation of a $\mathrm{BCl}$.

Among various electromagnetic signals that can be detected and utilized for $\mathrm{BCl}$ control, sensorimotor rhythm (SMR) is one of the most common. Sensorimotor rhythm-based $\mathrm{BCls}$ (SMR-BCls) (also referred to as motor imagery $\mathrm{BCls}$ - Ml-BCls) can detect the event-related desynchronization (ERD) in the electromagnetic signal recorded from sensorimotor areas of the brain during the motor imagery task. Figure 3 provides examples of motor imagery-related responses during a motor imagery task by using different signal recording modalities. SMR-BCls hold great potential for improving clinical outcomes in patients with compromised motor function. Indeed, the advancement of motor rehabilitation is the classic goal of SMR-BCl research. ${ }^{3} \mathrm{~A}$ comprehensive review of SMR-BCl studies suggests EEG-based SMR$\mathrm{BCl}$ intervention is a promising rehabilitation

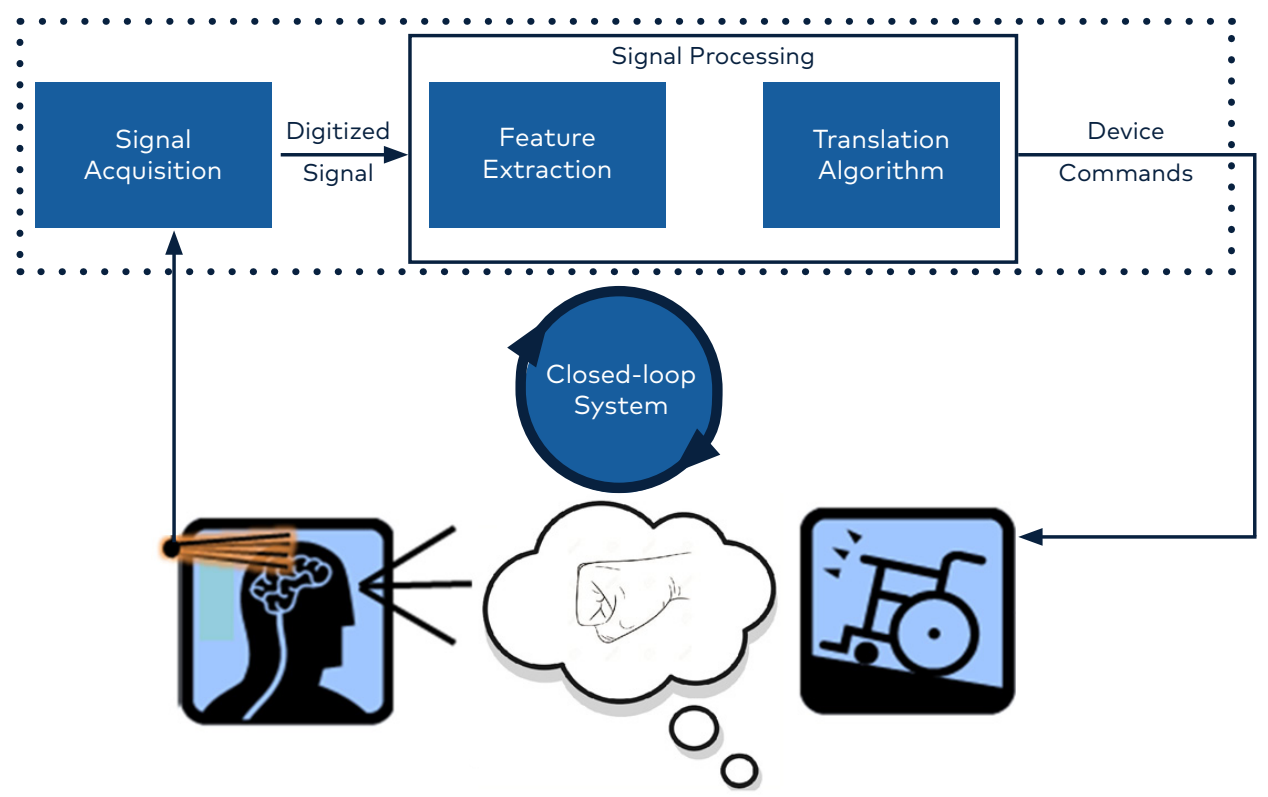

Figure 2. Brain Computer Interface $(\mathrm{BCl})$ system set-up. A task (for example, imagining closing and opening the hand) triggers specific brain activity within the $\mathrm{BCl}$ user (for example, event-related desynchronization) that is detected by EEG, MEG, or ECOG. BCl processes this acquired signal, extracting relevant features according to a predefined or "adaptive" algorithm. The $\mathrm{BCl}$ translates the detected features into a device command (for example, a forward wheelchair movement). Device commands commonly involve directional control. 


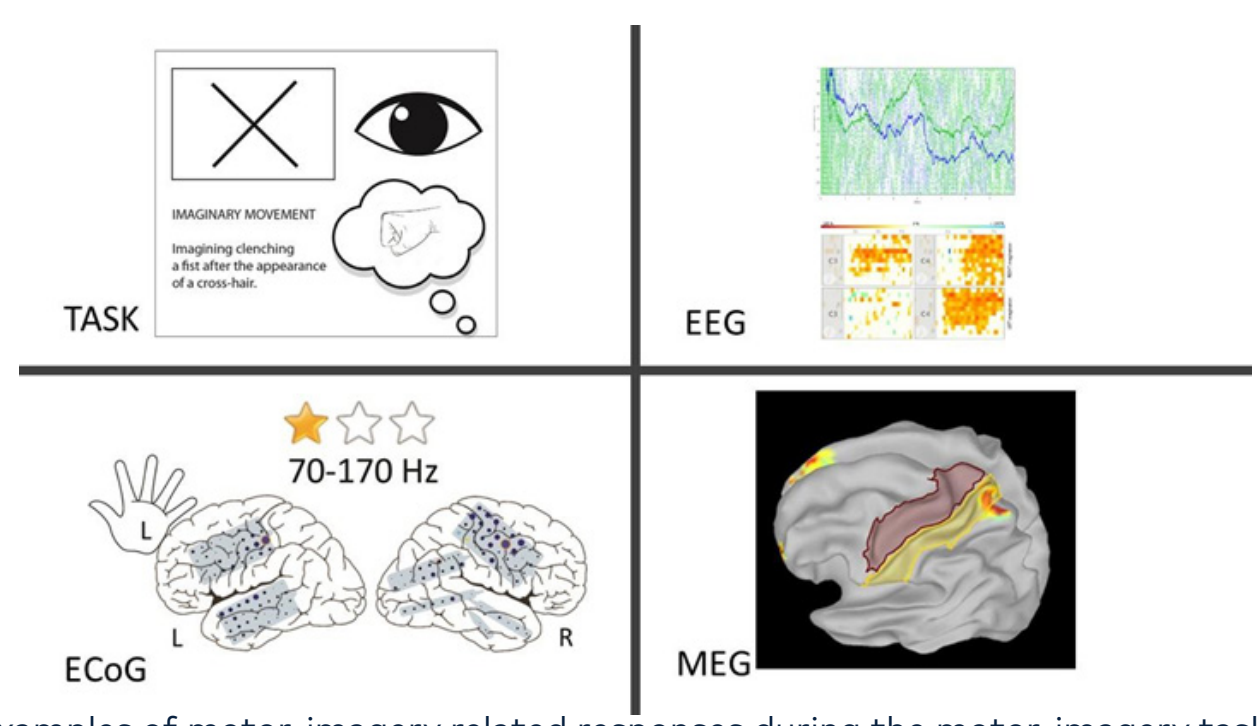

Figure 3. Examples of motor-imagery related responses during the motor-imagery task (upper left), recorded with different imaging modalities. Motor imagery is defined as "a mentally rehearsed task in which movement is imagined but not performed." Motor imagery tasks may include practicing making a fist, walking, or grasping an object. Motor imagery is associated with the generation of electromagnetic brain activity. This brain activity for $\mathrm{BCl}$ control can be recorded by a set of electrode arrays placed on the scalp when employing electroencephalography (EEG, upper right), by electrode grids placed directly on the cortical surface when utilizing electrocorticography (ECOG, lower left), as well as by a set of sensors when using magnetoencephalography (MEG, lower right). ${ }^{2}$ (Images courtesy of the authors.)

approach for upper motor function rehabilitation after stroke. ${ }^{4}$ Moreover, in individuals with compromised skeletal and/or motor system function (such as paralysis and amputation), a $\mathrm{BCl}$ may be used as a substitute to overcome functional deficit. ${ }^{5}$ Directional control is another common SMR-BCls application for the manipulation of a cursor on a screen used, for example, for the steering of a wheelchair ${ }^{6}$ or the control of a robotic neuroprosthesis. ${ }^{7,8}$

With continued development, a future becomes possible where $\mathrm{BCls}$ are found throughout the surrounding environment and utilized in everyday activities by both healthy users (e.g., for augmentation of existing function) and disabled users (e.g., for functional improvement or total replacement of function) alike. We can refer to such BCls as "ecological." To allow for such ecological SMR-BCl implementation, it is imperative to understand how SMR$\mathrm{BCl}$ performance is influenced by the user's environments: both internal and external. Indeed, the performance of a SMR-BCl is largely determined by the efficacy of the user, the $\mathrm{BCl}$ itself and the operational conditions. The importance of accounting for the effects of these factors is crucial for SMR-BCl performance optimization, and thus for the future proliferation of $\mathrm{BCl}$ use in a real-world ("ecological") context. For this article, internal variables are defined as those factors largely originating from within the SMR-BCl user. External variables, on the other hand, are identified as those elements that mainly reside within the SMR-BCl itself or exist beyond the SMR-BCl user. It should be noted that these working definitions of internal and external variables are simply operational and are used for this paper. Variations on these terms are found elsewhere. In some circumstances, internal and external variables, defined as such here, can be highly intertwined and used interchangeably, for example, distractibility (originating within the user) and distractors (originating outside the user). Due to the large number of internal variables for consideration, we have limited the scope of this review article to only focus on the effect of internal variables on SMR-BCl performance. We have also prepared a systematic review of the effect of external variables on SMR-BCl performance in a sister article. ${ }^{9}$

Multiple studies have attempted to mimic and isolate internal variables, which may affect any metric of SMR-BCl performance, such as signal information transfer rate (ITR), correct response rate (CRR), adoption rate, classification 
accuracy and reaching target accuracy., ${ }^{1,6-8,11-13}$ (for more details, see Table 1).

The goals of our current review paper are the following: (1) To summarize and critically evalvate the existing body of knowledge about the factors affecting $\mathrm{BCl}$ performance by critically examining the effects of internal variables on SMR-BCl; (2) To colligate main predictors of $\mathrm{BCl}$ "literacy"; as well as (3) To discuss limitations and propose further directions of "eco- logical" SMR-BCl research along with other possible factors that may or may not affect the SMR-BCls' performance when presented within an "ecological" real-world context.

\section{Internal Variables and Their Effect on SMR-BCI Performance}

In this article, we define internal variables as elements, that to the major extent, originate from within the SMR-BCl user. They include,

\begin{tabular}{|c|c|c|c|}
\hline $\begin{array}{l}\text { Internal } \\
\text { Variables }\end{array}$ & Referenced Studies & $\begin{array}{l}\text { Effect on BCI } \\
\text { Performance }\end{array}$ & Details \\
\hline $\begin{array}{l}\text { 1.1.1 Motor } \\
\text { Imagery } \\
\text { and Hand } \\
\text { Coordination }\end{array}$ & $\begin{array}{l}\text { Bian et al. (2018); }{ }^{14} \\
\text { Halder et al. (2011);15 } \\
\text { Hwang et al. (2009);16 } \\
\text { Mashat et al. (2019);17 } \\
\text { Scherer et al. (2015); }{ }^{18} \\
\text { Silva et al. }(2020)^{10}\end{array}$ & $\begin{array}{l}\text { Positive } \\
\text { effect }\end{array}$ & $\begin{array}{l}\text { Repetition of a simple motor imagery task can } \\
\text { substantially improve sensorimotor rhythm } \\
\text { generation. } \\
\text { Motor imagery task complexity is directly re- } \\
\text { lated to the degree of SMR-BCl performance } \\
\text { improvement. }\end{array}$ \\
\hline $\begin{array}{l}\text { 1.1.2 Attention } \\
\text { and Motivation }\end{array}$ & $\begin{array}{l}\text { Botrel and Kubler } \\
\text { (2019);19 } \\
\text { Cho et al. (2016);20 } \\
\text { Emami and Chau } \\
\text { (2018); } \\
\text { Friedrich et al. (2011); }{ }^{11} \\
\text { Geronimo et al. } \\
\text { (2016);22 } \\
\text { Guger et al. (2003);12 } \\
\text { Guger et al. (2015);23 } \\
\text { Guger et al. (2000);24 } \\
\text { Hammer et al. (2012);25 } \\
\text { Hammer et al. (2014);26 } \\
\text { Jeunet et al. (2016);27 } \\
\text { Kleih and Kübler } \\
\text { (2013);28 } \\
\text { Kleih et al. (2011);29 } \\
\text { Leeb et al. (2007);30 } \\
\text { Meng et al. (2018);31 } \\
\text { Nijboer et al. (2010) }{ }^{13}\end{array}$ & $\begin{array}{l}\text { Positive } \\
\text { effect }\end{array}$ & $\begin{array}{l}\text { The performance level of concentration } \\
\text { strength accounted for a proportion of SMR- } \\
\mathrm{BCI} \text { performance variation or insignificant } \\
\text { positive association. } \\
\text { Strong positive correlation between SMR-BCI } \\
\text { classification accuracy and the "challenge" and } \\
\text { "incompetence fear" motivational components. } \\
\text { Intrinsic motivation was not associated with } \\
\text { SMR-BCI performance in a consistent manner. } \\
\text { High fatigue level significantly impaired the } \\
\text { subjects' motor imagery EEG separatability. }\end{array}$ \\
\hline $\begin{array}{l}\text { 1.2.1 Quality of } \\
\text { Life }\end{array}$ & Nijboer et al. (2010) ${ }^{13}$ & No effect & $\begin{array}{l}\text { No significant relationship was observed be- } \\
\text { tween SEIQoL-DW scores and SMR-BCl count- } \\
\text { ing accuracies. }\end{array}$ \\
\hline 1.2.2 Mood & $\begin{array}{l}\text { Atassi et al. (2011);32 } \\
\text { Botrel and Kubler } \\
\text { (2019);19 } \\
\text { Dryden et al. (2005);33 } \\
\text { Jeunet et al. (2015);34 } \\
\text { Nijboer et al. (2010);13 } \\
\text { Patten et al. (2003);35 } \\
\text { Thomschewski et al. } \\
(2017)^{36}\end{array}$ & $\begin{array}{l}\text { Nature of an } \\
\text { association } \\
\text { unclear }\end{array}$ & $\begin{array}{l}\text { No significant relationship was observed be- } \\
\text { tween mood and SMR-BCl counting accuracy. } \\
\text { Strong predictive model based on a personality } \\
\text { profile. } \\
\text { Positive association between mood improve- } \\
\text { ment, the duration of the study and SMR-BCI } \\
\text { control mastery of confidence levels. } \\
\text { Relaxation trainings did not improve SMR-BCl } \\
\text { performance. }\end{array}$ \\
\hline
\end{tabular}


Table 1. Summary of Internal Variables Affecting BCI Performance. Cont'd.

\begin{tabular}{|c|c|c|c|}
\hline $\begin{array}{l}\text { Internal } \\
\text { Variables }\end{array}$ & Referenced Studies & $\begin{array}{l}\text { Effect on BCI } \\
\text { Performance }\end{array}$ & Details \\
\hline $\begin{array}{l}\text { 1.3 Neurophysi- } \\
\text { ological Signals } \\
\text { Other than } \\
\text { SMR }\end{array}$ & $\begin{array}{l}\text { Ahn et al. (2013);37 } \\
\text { Ang and Guan (2016); }{ }^{38} \\
\text { Azab et al. (2019); }{ }^{39} \\
\text { Bamdadian et al. } \\
\text { (2014);40 } \\
\text { Belwafi et al. (2019); }{ }^{41} \\
\text { Blankertz et al. (2010);42 } \\
\text { Dinares-Ferran et al. } \\
\text { (2018);43 } \\
\text { Gaur et al. (2019);44 } \\
\text { Grosse-Wentrup and } \\
\text { Schölkopf (2012);45 } \\
\text { Guan et al. (2019);46 } \\
\text { Joadder et al. (2019);47 } \\
\text { Olias et al. (2019);48 } \\
\text { Robinson et al. (2018);49 } \\
\text { Vidaurre et al. (2011);50 } \\
\text { Zhang and Wei (2019);51 } \\
\text { R. Zhang et al. (2015);52 } \\
\text { T. Zhang et al. (2016);53 } \\
\text { Y. Zhang et al. (2019) }{ }^{54}\end{array}$ & $\begin{array}{l}\text { Positive } \\
\text { effect }\end{array}$ & $\begin{array}{l}\text { Inverse relationship between simple reaction } \\
\text { time and information transfer rate. } \\
\text { Spectral or network properties of resting state } \\
\text { EEG activity are effective predictors of user's } \\
\text { SMR-BCl performance. } \\
\text { Adaptive and co-adaptive strategies may re- } \\
\text { duce the number of SMR-BCl users who cannot } \\
\text { achieve SMR-BCI literacy. } \\
\text { Novel particle swarm optimization algorithm } \\
\text { significantly decreased classification error rate } \\
\text { and number of channels compared to common } \\
\text { spatial pattern methods. }\end{array}$ \\
\hline
\end{tabular}

but are not limited to, the BCl user's psychological, behavioral and biological status, along with mental state. This section is an overview of studies that examine the effects of these internal variables on SMR-BCl performance (for summary, see Table 1).

The question of internal variables and their effect on SMR-BCl performance becomes a topic of important discussion when a "BCl literacy" phenomenon is considered. BCl literacy is loosely defined as the user's ability to operate a $\mathrm{BCl}$ successfully. BCl literacy may be quantified as a classification accuracy of at least $80 \% .^{12}$ However, values as low as $70 \%$ may be considered promising for the potential of future use. ${ }^{50,55}$ One of the earliest estimates demonstrated only $19.2 \%$ of subjects achieved SMR$\mathrm{BCl}$ literacy. ${ }^{12}$ Later, Blankertz et al. reported that 8 out of 14 (57\%) naive $\mathrm{BCl}$ users achieved a classification accuracy of at least $84 \% .12,55$ With the development of improved $\mathrm{BCl}$ interfaces and training paradigms, this proportion became greater. Several more recent estimates exist, claiming that on average roughly $75 \%$ of $\mathrm{BCl}$ users are SMR-BCl literate. $26,27,37,50$

Hammer et al. attempted to understand the phenomena of $\mathrm{BCl}$ illiteracy and performance variance amongst SMR-BCl users by identifying significant psychological predictors of SMR-BCI performance. ${ }^{25}$ These authors concluded that fine motor skills, information processing and concentration degree are significantly positively associated with SMR-BCl performance.

\subsection{Psychological and Behavioral $\mathrm{BCI}$ Users' Characteristics 1.1.1 Motor Imagery and Hand Coordination}

As motor imagery is a key concept associated with SMR-BCl, it is considered an important internal factor influencing SMR-BCl performance. Motor imagery is defined as "a mentally rehearsed task in which movement is imagined but not performed." ${ }^{6} 6$ Supplementary motor areas and the right middle gyrus are neural substrates of considerable motor imagery activity, task monitoring and working memory. Their activation implies the acquisition and recall of sensorimotor responses necessary for the operation of an SMR-BCl. ${ }^{15}$ High aptitude $\mathrm{SMR}-\mathrm{BCl}$ users demonstrate higher activation of the supplementary motor area during motor imagery and motor observation when compared to motor execution tasks. ${ }^{15}$

Repetition of a motor imagery task can significantly augment the performance of an SMR-BCI. Repetition can lead to considerable changes in sensorimotor rhythm generation, 
resulting in improved SMR-BCl classification accuracy. ${ }^{16}$ Furthermore, Scherer et al. noted the robustness of motor imagery practice's effect. The investigators described the capacity of individually adapted motor imagery task repetitions to improve SMR-BCl performance across a range of different tasks. ${ }^{18}$

The complexity of the motor imagery tasks may be associated with the user's SMR-BCl performance. For example, some studies have demonstrated a positive relationship between motor imagery task complexity and event-related desynchronization..$^{14,17}$ It is anticipated that this enhanced sensorimotor rhythm activity has the potential to contribute to improved SMR-BCl performance. Bian et al. demonstrated that trials with complex motor imagery tasks are associated with statistically significant improvement of SMR-BCl classification performance relative to trials with simple motor imagery tasks. ${ }^{14}$ In trials with a complex motor imagery task, SMR-BCl users' mean classification accuracy of alpha and beta-band power spectral density increased by $5.58 \%$ relative to trials with simple motor tasks. Moreover, the highest increase of SMR-BCl classification accuracy observed in a single subject was $20 \%$. Supporting these data, Bian et al. and Mashat et al. demonstrated an increase of up to $7.25 \%$ in SMR-BCl classification accuracy for a complex task relative to a simple task. ${ }^{14,17}$ These are encouraging results for the application of an $\mathrm{SMR}-\mathrm{BCl}$ in a complex, diverse real-world context filled with a variety of complex, simultaneously presented tasks.

\subsubsection{Attention and Motivation Attention}

In a comprehensive literature review, Jeunet et al. identified attention as a crucial aspect of SMR-BCl performance. ${ }^{27}$ A study by Geronimo et al. identified a significant positive association between attention and the SMR-BCI classification accuracy of patients with amyotrophic lateral sclerosis (ALS). ${ }^{22}$ The investigators assessed the participants' attention capacity according to the ALS-cognitive behavioral scale. Attention was one of the four components of cognition in this scale. In particular, the attention domain was an important predictor of motor imagery quality. Quality was defined as the motor imagery signal robustness for a given electrode channel as calculated by the standard difference between the average power spectrum in left and right motor imagery trials. Attention, as a component of overall cognition, could lead to an increase in signal fidelity of task-relevant EEG band power. ${ }^{22}$ Botrel and Kubler further demonstrated that attention, defined as the ability to concentrate, is a significant predictor of SMR-BCl classification accuracy. ${ }^{19}$ Supporting this assertion, more sophisticated virtual cursor control via SMR-BCl was achieved when the modulation of endogenous visuospatial attention was enabled for $\mathrm{BCl}$ study participants compared with similar trials without endogenous visuospatial attention. ${ }^{31}$

Although we describe distractors as an external variable in another SMR-BCI review article, its relationship to attention makes it relevant for discussion at this time. ${ }^{9}$ We concluded that distractors have a significant positive effect on SMR-BCl performance. ${ }^{9}$ This conclusion was supported by the finding that passive auditory distraction optimized mental imagery-based $\mathrm{BCl}$ classification accuracy. Additionally, intermittent small visual distractors altered mu and beta power of motor imagery-specific patterns but did not significantly alter SMR-BCl classification accuracy.

Distraction may be considered a state of the absence of attention. For this reason, it is anticipated that distraction is inversely related to SMR-BCl performance. Friedrich et al. demonstrated that auditory distractors had no adverse effect on cue-guided 4-class hybrid P300-SMR-BCl performance. ${ }^{11} \mathrm{BCl}$ performance was maintained during auditory distractors in all mental tasks. Emami and Chau further explored the influence of distractors with a study of the relationship between visual distractors and SMR-BCl classification accuracy. ${ }^{21}$ Infrequent, small visual distractors altered mu and beta power of motor imagery-specific patterns but did not significantly alter SMR-BCl classification accuracy. Participants achieved a mean classification accuracy of 81.5 $\pm 14 \%$ for non-distractor trials, and $78.3 \pm 17 \%$ for distractor trials. ${ }^{21}$ These developments are promising for the everyday application of $\mathrm{BCls}$ in noisy real-world contexts.

Earlier studies identified varying relationships between attention and SMR-BCI performance. 
The user's concentration strength or degree of sustained attention, as measured by the Attitudes Towards Work test variable "performance level," accounted for approximately $19 \%$ of the variance in SMR-BCl performance. ${ }^{25}$ Notably, a different study revealed a positive yet insignificant association between the predictive value of concentration ability and SMR$\mathrm{BCl}$ performance. ${ }^{26} \mathrm{~A}$ possible explanation for this discrepancy is that different metrics were used in these studies. Whereas the 2012 study by Hammer et al. used performance-based metrics to assess sustained attention, the 2014 research by Hammer et al. used self-reported metrics for the same purpose. ${ }^{25,26}$ By virtue of its relationship with attention, motivation has the potential to influence SMR-BCl performance as well as to directly influence the subjects' attention towards the task at hand.

\section{Motivation}

Nijboer et al. defined motivation as "an impetus toward a goal for all current processes" and quantified it with a modified version of the Questionnaire for Current Motivation (QCM). ${ }^{13}$ With the QCM, subjects self-evaluated their current motivation according to a Likert-type score of four internal motivational factors: mastery confidence, incompetence fear, challenge and interest. Their results did not reveal a clearly defined overall correlation between motivation and SMR-BCl performance. This led to the conclusion that motivational factors may affect SMR-BCl performance on an individual, case by case basis. ${ }^{13}$

Importantly, Nijboer et al. cautioned against the extrapolation of their study results in clinical patients to the general population. ${ }^{13}$ The authors suspected that a different relationship would exist between the motivation of healthy users and SMR-BCl performance than the one determined in their study with clinical patients. Indeed, the study by Leeb et al. identified a strong positive correlation between the motivation or mental effort of ten healthy users and their SMI-BCl performance. ${ }^{30}$ These findings are supported by Kleih et al., who observed a positive correlation between SMR-BCl classification accuracy and the "challenge" and "incompetence fear" motivational components of 41 healthy subjects. ${ }^{29}$ This is consistent with other sources suggesting that motivation has been identified to have a significant positive correla- tion with SMR-BCl classification accuracy. ${ }^{23,24}$

It should be noted that the study by Nijboer et al. only contained ALS patients, no healthy subjects..$^{13}$ As opposed to healthy study participants, the ALS patients have a vested interest in the treatment and management of their condition. This deep awareness was reflected in the ALS patients' QCM scores, which demonstrated that the patients were highly intrinsically motivated. ${ }^{13}$ Healthy individuals could, however, be extrinsically motivated (for example, by being provided with monetary compensation). Monetary compensation is a common practice to encourage subject participation. Offering a financial incentive would moderately provide extrinsic motivation for subjects to become involved with the study. ${ }^{28}$

Mediating factors can influence the performance of $\mathrm{BCls}$. The presence of mediating factors may explain the discrepancy in users' SMR-BCl performance by Guger et al. ${ }^{12,23}$ In 2015, Guger et al. reported significantly higher SMR-BCl performance metrics than those of Guger et al. in 2003.12,23 Motor imagery experiments were conducted with recoveriX-a BCl system for stroke rehabilitation. Further details on recoveriX are provided in Figure 4.

Five patients post-stroke (ages: 40, 61, 63, 66, 68) were trained with left and right motor imagery paradigms in 30 minute sessions. ${ }^{23}$ When the $\mathrm{BCl}$ detected a brain response associated with imaginary hand/arm movement, a functional electrical stimulator was triggered to produce a real hand/arm movement. All five patients reached a very high $\mathrm{BCl}$ accuracy of 96 , 96, 96, 98 and $99 \%$ within 25 training sessions. Recently, Cho et al. performed a similar motor imagery experiment involving recoveriX with one stroke patient. ${ }^{20}$ Similarly to the previous study, this patient achieved a very high $\mathrm{BCl}$ performance accuracy of $96 \%$ within only 10 training sessions. One possible explanation for this variation in the number of training sessions needed to achieve high $\mathrm{BCl}$ performance accuracy is that the latter study only had a single patient. This individual may not have represented the average or normal user's SMR-BCl performance.

According to Guger et al. (2015), an important factor for such high $\mathrm{BCl}$ performance accura- 


\section{HCA Healthcare Journal of Medicine}

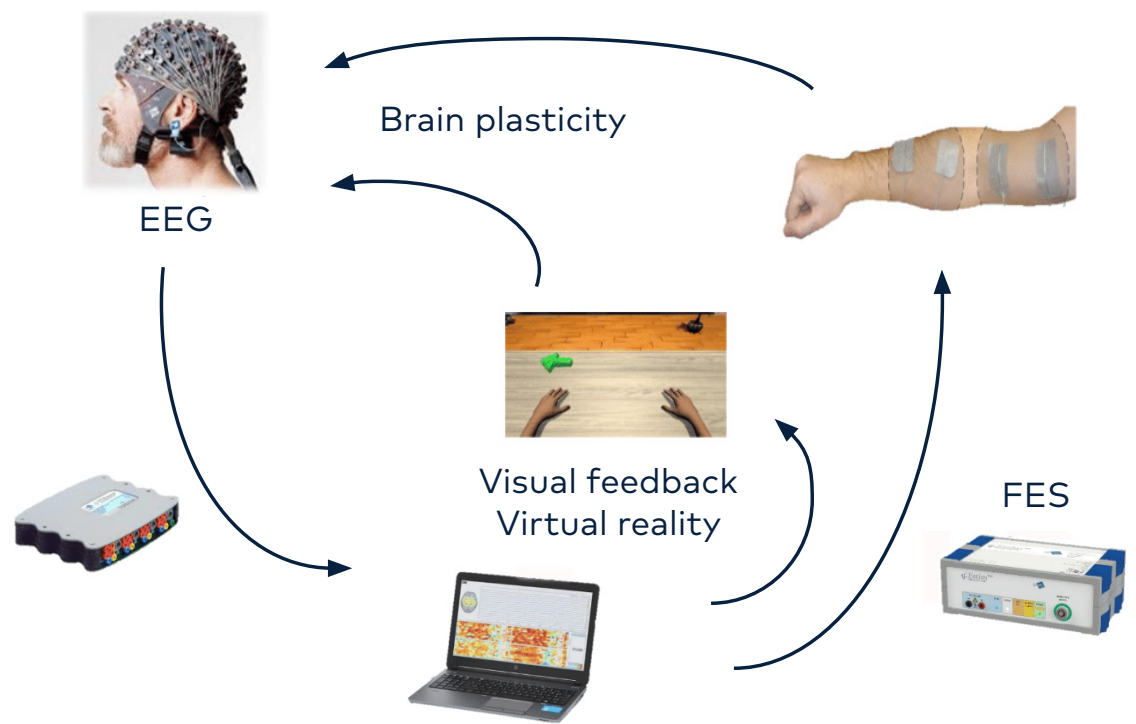

Figure 4. SMR-BCl system (recoveriX) for motor recovery in stroke patients. This complete hardware and software platform that is capable of recording and analyzing the EEG for rehabilitation consists of: an electroencephalography system (EEG), an avatar ("virtual reality") and a functional electrical stimulation (FES). The system provides the real-time, monitor-based virtual reality feedback (with an avatar and real-time brain activation maps). (Photographs courtesy of the authors.)

cies is the patients' motivation to participate in the training to improve their motor functions. ${ }^{23}$ In their 2000 study, Guger et al. demonstrated that healthy controls can reach high classification accuracies within 6-7 training sessions of about 30 minutes. ${ }^{24}$ In fact, three healthy students tested in this study achieved $\mathrm{BCl}$ performance accuracies above 95\%. One subject even performed the first trial of $100 \%$ classification accuracy of all BCl studies. ${ }^{24}$ The physical status of the participants of the 2015 Guger et al. study and the 2000 Guger et al. study served as a study in contrasts. ${ }^{23,24}$ In the later study, 3 highly motivated students were selected to achieve these results. On the other hand, the earlier study involved recovering stroke patients. These SMR-BCl performance findings in a diverse patient population of highly motivated healthy subjects and afflicted patients offer promise for a higher future SMR-BCl adoption rate.

We will consider mental fatigue as the absence or diminution of motivation. Subjects with higher motivation should be able to delay the influence of mental fatigue. Conversely, subjects with lower motivation may prematurely succumb to mental fatigue's influence. It is anticipated that an indirect relationship would exist between mental fatigue and SMR-BCl performance. Indeed, Talukdar et al. supported this assumption. ${ }^{57}$ The investigators monitored mental fatigue in eleven participants over the course of prolonged motor imagery sequences. High fatigue level significantly impaired the subjects' motor imagery-related EEG signal disctrimination. ${ }^{57}$ The clear interpretation of EEG signals is crucial to optimal BCl performance. It is anticipated that decreased motor imagery-related EEG signal discrimination should interfere with the ability of an SMR-BCl to translate neural activity into motor machine commands. Future research is needed to confirm the correlation between decreased motor imagery-related EEG discrimination and SMR-BCl performance. These findings offer a potential electrophysiologic mechanism for decreased SMR-BCl performance with decreased motivation, as considered by mental fatigue.

\subsection{Psychological Variables and Mental State \\ 1.2.1 Motor Imagery and Self- Prediction of the SMR-BCI Competency}

Formal analysis of psychological variables and mental state has attempted to support the assumption that these internal variables influence SMR-BCl performance. Spatial ability is associated with motor imagery, and therefore, potentially with the SMR-BCl performance. The men- 
tal exercise of motor imagery facilitates neural network plasticity across several regions of the brain, thus developing spatial ability. ${ }^{58}$ It was found that fine motor skills and the accuracy of information dissemination were responsible for $11 \%$ of SMR-BCl performance variance..$^{25}$ In addition to the effect on SMR-BCl variance demonstrated by the 2012 Hammer et al. study, the 2014 study by Hammer et al. confirmed the predictive role of visuo-motor coordination ability for SMR-BCI performance..$^{25,26}$

Furthermore, Ahn et al. described that self-prediction of SMR-BCl competency in subjects with SMR-BCl experience shares a statistically significant relationship and moderate correlation with SMR-BCl performance. ${ }^{59}$ Subjects' self-prediction of SMR-BCl competency improved over the course of repeated trials, even without feedback information. ${ }^{59}$ In a later study, Rimbert et al. highlighted the limitations of self-prediction. ${ }^{60} \mathrm{~A}$ subjective motor imagery questionnaire failed to predict the SMR-BCl performance of 35 healthy subjects.

Nijboer et al. tried to isolate several key internal variables that may affect SMR-BCl. ${ }^{13}$ In particular, these researchers evaluated the influence of quality of life and mood on the performance of SMR-BCls. Subjects were asked to control the vertical movement of a cursor in order to hit a target. The authors assessed SMR-BCI performance as a correct response rate (CRR), defined as the percentage of hit targets in a single session. Subjects used a $6 \times 6$ character matrix to copy the text of the sentence, "Franz chases in a completely shabby taxi across Bavaria." (This sentence in German is comprised of every letter of the alphabet, "Franz jagt im komplett verwahrlosten Taxi quer durch Bayern." 13 and serves as a German analogue of the English alphabet-containing phrase "The quick brown fox jumps over the lazy dog."). In order to compare CRR, the chance level of hitting a target $(1 / 2=0.5)$ or select a correct character $(1 / 36=0.027)$ must be considered. To standardize for chance, CRR was calculated into an information transfer rate (ITR). The findings reported by Nijboer et al. are discussed in the following subsections. ${ }^{13}$

\subsubsection{Quality of Life}

Quality of Life (QoL) provides a framework within which SMR-BCl training and implemen- tation occurs. As a result, Nijboer et al. contend that this context may inform SMR-BCl performance and was the first to explore the QoL and $\mathrm{MI}-\mathrm{BCl}$ performance relationship. ${ }^{13}$ In their study, the authors used the Schedule for the Evaluation of Individual QoL Direct Weighting (SEIQoL-DW) to measure the subjects' QoL.

Before completing the SMR-BCl portion of the study, all subjects demonstrated a QoL ranging from satisfactory to good (average SEIQoLDW score before SMR training: 76.6). The results did not demonstrate a significant relationship between QoL and SMR-BCI performance. SMR-BCl performance accuracies were within the normal range even in those subjects who noted QoLs below average, further indicating that QoL may not have influence on SMR-BCl performance.

\subsubsection{Mood}

According to Nijboer et al., mood affects cognitive function..$^{13}$ Mood's influence on cognition leads to anticipation that subjects with a better mood would be more receptive to SMR-BCI training. In turn, it can be expected that mood would demonstrate a positive correlation with SMR-BCl performance. However, after evaluating the change in subjects' psychological state as they went through the SMR-BCl training and actual SMR-BCl control process, Nijboer et al. observed no relationship between mood and SMR-BCl performance. ${ }^{13}$ Interestingly, the results showed an association between mood improvement and the duration of the study. The authors suggested that the reason behind this improvement in mood might due to the decrease in SMR-BCl control incompetence levels with the progression of the experiment. This change was accompanied by a corresponding increase of confidence levels in SMR-BCl control mastery, thus improving the mood of study participants. ${ }^{13}$

Botrel and Kubler supported the mood findings of Nijboer et al. ${ }^{13,19}$ Four 30-minute relaxation trainings prior to a SMR-BCI session failed to improve the participants' SMR-BCl performance relative to groups who received one or no relaxation session.

The relationship between depression and SMR$\mathrm{BCl}$ performance is highly relevant as disabled $\mathrm{SMR}-\mathrm{BCl}$ users, due to their limited physical condition, frequently battle depression. ${ }^{32,33,35}$ 
Similarly to the results demonstrating no statistically significant effect of mood on SMR$\mathrm{BCl}$ performance, Nijboer et al. showed no clear relationship between depression and subjects' SMR-BCl performance..$^{13}$ Later research supported this uncertain association. ${ }^{36}$ In a study involving seven male patients with traumatic spinal cord injury, two patients demonstrated Beck Depression Inventory scores consistent with depression. These two patients reported the most problems with movement imagination, but statistical analysis could not confirm an association between depression and decreased SMR-BCI performance across all healthy controls and patients. ${ }^{36}$

In contrast to the findings of Nijboer et al., Jeunet et al. developed a strong predictive model for SMR-BCl performance based on the user's mood. ${ }^{13,34}$ Through the use of a psychometric questionnaire, Jeunet et al. determined a personality profile based on moods, traits and emotional states. ${ }^{34}$ More studies are needed to clarify the exact nature of the relationship between mood and SMR-BCl performance. Mood's effect on SMR-BCl performance is still not well understood. In the application of the Nijboer et al. findings, caution would be warranted. $^{13}$

\subsection{Neurophysiological Signals Other than SMR}

Current literature suggests that physiological signals can be used to predict users' SMR-BCl performance. 37,38,40,42,45,47,49,50,52,53,61-63 For example, Grosse-Wentrup and Schölkopf could forecast subjects' inter-trial SMR-BCl classification accuracy by calculating the measured differences in gamma-power between two fronto-parietal networks. ${ }^{45}$ These networks correlated with fMRI-identified neurological sites of focused attention and working memory, suggesting gamma oscillations are the neurophysiological signal correlate of these cognitive processes. ${ }^{45}$

Darvishi et al. identified simple reaction time as a significant predictor of subjects' future SMR$\mathrm{BCl}$ performance. ${ }^{61}$ Participants demonstrated an inverse relationship between simple reaction time and information transfer rate. In addition, researchers observed alpha and beta-wave activity of greater amplitude in this same participant population. ${ }^{51}$
However, a controversy exists regarding the effect of background electrophysiological brain activity on SMR-BCl performance. Bamdadian et al. used pre-cue EEG rhythms from different areas of the brain to develop a novel coefficient for predicting SMR-BCl classification performance. ${ }^{40}$ Incorporating both spatial and spectral EEG signal information, Bamdadian et al. used this coefficient to predict users' SMR$\mathrm{BCl}$ classification accuracy. ${ }^{40}$ The results of this study suggested that users' higher frontal theta and lower posterior alpha activity led to improved SMR-BCl classification values. Contrary to observations by Bamdadian et al., a study by Ahn et al. described a moderately to strongly significant positive association between users' high theta and low alpha power with respect to SMR-BCl illiteracy. ${ }^{37,40}$ Robinson et al. further explored the ability of resting state activity to predict SMR-BCl performance. ${ }^{49}$ The results of their study suggested that entropy and gamma power from pre-motor and posterior areas as well as beta power from centro-parietal areas have a strong predictive correlation with SMR$\mathrm{BCl}$ performance. ${ }^{49}$

Investigators have proposed alternative predictive elements of SMR-BCl performance. ${ }^{38,42,50,52}$ Zhang et al. identified a strong correlation between the spectral entropy of eyes-closed resting-state EEG activity with inter-session SMR-BCl performance. ${ }^{52}$ In particular, these authors selected the $\mathrm{C} 3$ channel as a potential biomarker of SMR-BCl performance. The findings demonstrated $89 \%$ effectiveness of an inter-session spectral entropy to predict the average SMR-BCl classification accuracy. ${ }^{52}$ Zhang and Wei explored the role of channel selection on SMR-BCl performance..$^{51}$ Experimental results revealed that a novel particle swarm optimization algorithm significantly decreased classification error rate and the number of channels compared to common spatial pattern methods, which had previously demonstrated great promise..$^{51,54}$

In a different study, Zhang et al. associated the resting-state EEG network with SMR-BCl performance. ${ }^{52}$ Efficient resting-state network EEG activity qualities, such as greater mean functional connectivity, node degrees and edge strength led to enhanced user SMR-BCl performance. Conversely, increased characteristic path length was associated with decreased 
user SMR-BCl performance. Characteristic path length is defined as "the average shortest path length between all pairs of nodes in the network". ${ }^{52}$

In addition, Blankertz et al. proposed a neurophysiological predictor of SMR-BCl performance. ${ }^{42}$ The researchers derived this neurophysiological predictor from a two-minute recording of a "relax with eyes open" condition using two Laplacian EEG channels. This study observed only a moderately significant positive correlation between this prognostic technique and $\mathrm{BCl}$ literacy. ${ }^{42}$

Moreover, Ang and Guan determined an EEGbased adaptive strategy to reduce the variance between the SMR-BCl classification accuracies of calibration and feedback sessions. ${ }^{38}$ In the adaptive strategy, a subject-specific model is continuously developed during these sessions based on EEG signals. This subject-specific model more accurately interprets users' EEG signals, thus improving SMR-BCI performance. ${ }^{38}$

Further studies support this adaptive strategy approach..$^{39,41,43,44,46-49}$ For instance, Joadder et al. developed a subject-independent performance-based EEG feature fusion algorithm in combination with machine learning for the classification of motor imagery signals into certain states. ${ }^{47}$ This novel approach yielded a classification accuracy of $99 \%$.

Prolonged calibration time is a barrier to widespread SMR-BCl use. Gaur et al. proposed an adaptive strategy of tangent space features-based transfer learning classification model for SMR-BCls to eliminate lengthy training sessions. ${ }^{44}$ The researchers defined transfer learning as "the process of applying the knowledge gained from one task to another related activity". ${ }^{44}$ Expanding on a subject-specific multivariate empirical mode decomposition model, the researchers identified shared structures of the tangent space features among participants. This model was then used to evaluate the SMR-BCl classification accuracy of unseen trials. This novel tangent space features-based learning classification model yielded a similar SMR-BCl classification accuracy to other current adaptive classifiers such as subject-specific multivariate empirical mode decomposi- tion-based filtering method, common spatial patterns on band-pass filtered EEG between $8 \mathrm{~Hz}$ and $30 \mathrm{~Hz}$ with linear discriminant analysis, common spatial patterns with covariate shift detection and adaptive learning, as well as filter bank common spatial pattern. Thus, the adaptive strategy of transfer learning techniques can be used to mitigate the problem of time-intensive training sessions. ${ }^{44}$

Olias et al. improved the widely used standard power normalization technique of EEG preprocessing through two new methods. ${ }^{48}$ First, researchers presented a novel power-normalizing technique that is scaled independently of the observation trials. Second, the investigators proposed the application of an alternative shrinkage covariance matrices estimate that is based on normal statistical features. Together, these two methods yielded a significant improvement in SMR-BCl classification results. ${ }^{48}$

Co-adaptive SMR-BCl calibration advances this concept further, wherein both the algorithm of the SMR-BCl and the mental strategy of the user are mutually trained..$^{50}$ Co-adaptive SMR-BCl calibration has the potential to extend SMR-BCl literacy to new users. ${ }^{50}$ With a co-adaptive SMR-BCl, naive users may be trained to operate an SMR-BCl within minutes of the first session. Moreover, SMR-BCl users, who previously failed to achieve adequate SMR-BCl control with an adaptive strategy, gained SMR-BCl literacy after fifteen minutes of feedback after the first run. These users demonstrated an improvement of SMR-BCl performance both during a session and between the first and last run. ${ }^{50}$

Overall, there is evidence that physiological signals are an effective predictor of users' SMR$\mathrm{BCl}$ performance. Therefore, SMR-BCl candidate screening tools may include measures of their resting state activity, such as spectral or network properties, which would further the overall goal of widespread SMR-BCl application in everyday life by more readily recognizing those users of greater potential to adopt this technology successfully. Beyond the identification of potential SMR-BCl users, adaptive and co-adaptive SMR-BCl calibration strategies may reduce the number of SMR-BCl users who cannot achieve SMR-BCl literacy. Together, predictive biomarkers and adaptive strategies can expand the potential SMR-BCl user base. 


\section{Limitations and Future Perspectives}

The study of the effects of internal variables on SMR-BCl performance is incomplete. Limitations exist within the previously described studies, and opportunities for future perspectives and development persist. In spite of the previously described motor imagery practice approach used to improve SMR-BCl performance, there remain users who are SMR-BCl "illiterate." Indeed, according to a study by Jeunet et al., only $70-90 \%$ of users are able to achieve SMR-BCl literacy. ${ }^{27}$ Importantly, these authors have demonstrated that standard SMR-BCl training is insufficient for the SMR-BCl literacy improvement because it lacks adequate testing of spatial ability. ${ }^{64}$ Spatial ability (such as two-hand coordination, sports or music practice) is an important factor of a successful SMR-BCl performance. The development of this aptitude is a significant component of an effective SMR-BCl training paradigm. ${ }^{64}$ More research is needed to elucidate a motor imagery practice approach with a more effective spatial ability component.

In addition, the effect of motor imagery practice on SMR-BCl performance may be outpaced by simple motor observation. Halder et al. noted that brain function during motor observation could predict SMR-BCl user proficiency. ${ }^{15}$ This finding is further supported by the higher number of activated voxels in the right middle frontal gyrus during motor observation rather than motor imagery or motor execution..$^{15}$ The effect of motor observation and its relationship with motor imagery are areas of future perspectives for the influence of motor imagery on SMR-BCl performance.

In the study by Nijboer et al., the authors identified several study limitations and areas for further inquiry. ${ }^{13}$ The small $(n=6)$ study sample limited the significance of the findings. Furthermore, a larger testing population would allow for more demographic diversity to facilitate further inquiry into the relationship between numerous internal factors and SMR-BCI performance.

In addition, a larger sample size would allow for the incorporation of healthy subjects to serve as a control. ALS patients often have large electromyographic (EMG) artifacts because they cannot cease the symptoms of their con- dition such as coughing, swallowing or yawning during $\mathrm{BCl}$ experimental trials. These interrupted $\mathrm{BCl}$ trials have a low signal-to-noise ratio. This complicates the interpretation of study results because it is difficult to discern the signal of interest from the confounding signals. This factor of low signal-to-noise ratio can be controlled for with the presence of healthy subjects. Healthy subjects do not suffer from the described ALS-related symptoms and do not interrupt the $\mathrm{BCl}$ trials with the same regularity. As a result, healthy subjects demonstrate a higher signal-to-noise ratio than their ALS counterparts. The high signal-to-noise ratio of healthy control subjects would elucidate the SMR results of ALS patients, and thus would facilitate meaningful analysis. ${ }^{13}$

Nijboer et al. indicated that the influence of incentives on extrinsic motivation and SMR-BCl performance is another future area of research..$^{13}$ Healthy subjects may provide a wider range of QCM motivational scores than the intrinsically motivated ALS patients. The incorporation of healthy subjects would allow for a QCM data set with greater variance. This would facilitate the investigation into the impact of incentives on extrinsic motivation and SMR-BCI performance.

Conflicting evidence by Bamdadian et al. and Ahn et al. exists describing the nature of the relationship between alpha and theta electroencephalographic waves with SMR-BCl performance. ${ }^{37,40}$ One possible explanation for this inconsistency is the locations of the neurophysiological recording sites, where the signal was sampled. For example, Bamdadian et al. selected frontal and parietal areas to calculate theta and alpha activity respectively. ${ }^{40}$ On the other hand, Ahn et al. examined the prefrontal and central areas for theta activity. ${ }^{37}$ Alpha activity was most strongly present in the occipital area. However, these sites may not fully explain the different findings in these two studies. More research is needed to clarify this question.

\section{Next Steps}

The domain of SMR-BCl performance optimization involves the SMR-BCl users. While not all variables have demonstrated a positive effect, internal variables have the potential to improve SMR-BCl performance metrics such as classification accuracy, information transfer 
rate or task duration. Gaps of knowledge remain that may or may not affect the real-world application of SMR-BCl. Sample size is one of the most crucial aspects towards producing significance in a study. Here are some suggestions to investigate the effect of internal variables further:

\subsection{Training Paradigm Development}

During SMR-BCl training, subjects may become bored and frustrated with the repetitive nature of a simple task. As a result, the subjects' attention may wane during SMR-BCl training. $\mathrm{Ni}-$ jboer et al. suggested that an optimal training paradigm may involve a stepwise, progressively more complex task to maintain the selective and sustained attention of the subjects. ${ }^{13}$ The exact nature of this increasingly complex task and the related protocols for it are another area that Nijboer et al. suggested for future research. ${ }^{13}$

\subsection{Medication}

Interestingly, Meng et al. explored the influence of caffeine consumption on resting state EEG and SMR-BCl performance..$^{62}$ Although caffeine consumption substantially decreased alpha and beta-band power in 26 healthy subjects, the researchers found no evidence of significant change on subjects' SMR-BCl performance relative to controls who did not consume caffeine. ${ }^{62}$ Moreover, sugar consumption did not significantly influence either EEG resting state activity or SMR-BCl performance. ${ }^{62}$ The relationship between frontal EEG activity and SMR-BCl performance has been further investigated by Zhang et al., who showed that subjects with an efficient fronto-parietal attention network activity perform better on SMR-BCl. ${ }^{53}$

Locked-in patients presently comprise many SMR-BCl users. Locked-in patients often suffer from conditions such as ALS, multiple sclerosis (MS) or spinal cord injury. As part of their treatment plan, SMR-BCl users may be prescribed antidepressants, opioids or benzodiazepines. Medical professionals must be aware of not only how medications affect not only their patients' physical being, but also their patients' ability to communicate with the world around them. Nijboer et al. suggested that a next step in the field of SMR-BCl research is an investigation into the effect of medications on SMR-BCl performance..$^{13}$

\subsection{Gender and Education}

The gender of a user has been demonstrated to influence the classification accuracy of an SMR$\mathrm{BCl}$. Cantillo-Negrete et al. revealed that a gender-specific subject-independent design led to a significantly greater SMR-BCl performance than the performance observed in an SMR-BCl where gender is not considered. ${ }^{65}$ Subject-independent design focuses on achieving $\mathrm{BCl}$ literacy while reducing SMR-BCl training requirements in the interest of the patient population who cannot meet this demand. For subject-independent design, researchers identify Common Spatial Patterns and log variance features amongst a group of subjects. Cantillo-Negrete et al. classified these data amongst two groups, males and females. ${ }^{65}$ The investigators tasked both healthy subjects and stroke patients with imagining the opening and closing of the left and right hands. In almost all of the experimental conditions, the gender-specific $\mathrm{SMR}-\mathrm{BCl}$ designs were associated with greater performance. However, the improved classification accuracy observed with a gender-specific $\mathrm{SMR}-\mathrm{BCl}$ design was not always associated with the intended gender of the user. ${ }^{65} \mathrm{~A}$ user's gender may influence the performance of an $\mathrm{SMR}-\mathrm{BCl}$, but more research is needed to more clearly elucidate this relationship.

The future goal of SMR-BCl use is for the widespread adoption of SMR-BCls amongst all peoples. Ideally, no barriers for use would exist. Education is one potential barrier for SMR$\mathrm{BCl}$ use. Education may be inversely related to comprehension of difficult instructions. As an emerging technology, SMR-BCl setup and operation involves numerous steps with sophisticated technologies. For this reason, it is anticipated that those who struggle to accurately operate the brain-computer interface may not experience optimal SMR-BCl performance. Moreover, Skrandies and Klein demonstrated a significant association between successful learning divisibility rules and the changes in frequency of task-related EEG. ${ }^{66}$ This neurophysiological modulation may facilitate signal acquisition for SMR-BCl performance. Education is the repetition of learning for the development of a broad base of knowledge. Repetition of a motor imagery task improves SMR-BCl performance. ${ }^{16}$ We propose that repetition of learning may facilitate the generation of optimal SMR patterns for the operation of an SMR-BCl. 


\section{Conclusions and Future Perspectives}

SMR-BCI holds great potential for widespread application of both healthy and physically limited patients. The goals of our current review paper were (1) to compile established literature about the effects of internal variables on SMR$\mathrm{BCl}$ performance, (2) to identify predictive biomarkers of $\mathrm{BCl}$ aptitude and (3) to identify limitations and propose further perspectives of "ecological" Ml-BCl research.

This review article is intended to serve as an overview of studies that examine the effects of internal variables on SMR-BCl performance. We may conclude that attention, motivation and neurophysiological signals other than SMR share significantly positive relationships with $\mathrm{BCl}$ performance. Conversely, quality of life and mood do not have any clear association with SMR-BCl performance. A comprehensive literature review yields several main predictors of SMR-BCl literacy: simple reaction time, spectral and network properties of resting state activity, adaptive strategies and co-adaptive strategies. The identification of biomarkers of effective SMR-BCl control helps to identify prospective candidates for SMR-BCl. Additional biomarkers would provide a more selective and sensitive screening tool for potential SMR$\mathrm{BCl}$ users. More research is needed to identify additional biomarkers. For more details, please reference Table 1.

Due to the limited availability of this emerging technology, sample size has been a recurring concern for SMR-BCl research. More subjects would allow for the discovery of relationships with greater significance, the introduction of healthy controls and further investigation of additional variables. We proposed next steps for the SMR-BCl research with respect to internal variables. More research is needed to describe the influence of gender and education.

\section{Abbreviations}

ALS - amyotrophic lateral sclerosis; BCls brain-computer interfaces; CRR - correct response rate, CVEP- code-modulated visual evoked potentials; EEG - electroencephalography, EMG - electromyography, ERD - event-related desynchronization, ERPs - event-related potentials; ITR - information transfer rate; $\mathrm{MI}$
- motor imagery, MIT - motor imagery task without feedback, MS- multiple sclerosis; QCM - questionnaire for current motivation, QoL quality of life; SEIQoL-DW - schedule for the evaluation of individual QoL direct weighting, SMR - sensorimotor rhythm; VEP - visual evoked potential

\section{Conflicts of Interest}

Dr. Christoph Guger is the CEO and owner of g.tec, a company that sells neurotechnology on the international market.

Drs. Horowitz and Korostenskaja declare they have no conflicts of interest.

Dr. Horowitz is an employee of University of Central Florida/HCA Healthcare GME Consortium, an organization affiliated with the journal's publisher.

This research was supported (in whole or in part) by HCA Healthcare and/or an

HCA Healthcare affiliated entity. The views expressed in this publication represent those of the author(s) and do not necessarily represent the official views of HCA Healthcare or any of its affiliated entities.

\section{Author Affiliations}

1. Functional Brain Mapping and Brain Computer Interface Lab, Neuroscience Institute, AdventHealth Orlando, Orlando, FL, USA

2. g.tec Medical Engineering $\mathrm{GmbH}$, Graz, Austria

3. MEG Lab, AdventHealth for Children, Orlando, FL, USA

4. Department of Psychology, College of Arts and Sciences, University of North Florida, Jacksonville, FL, USA

5. Comprehensive Epilepsy Center, AdventHealth Orlando, Orlando, FL, USA

6. University of Central Florida/HCA Healthcare GME Consortium, Gainesville, Florida

\section{References}

1. Guger C, Daban S, Sellers E, et al. How many people are able to control a P300-based brain-computer interface (BCl)?. Neurosci Lett. 2009;462(1):94-98. https://doi.org/10.1016/j. neulet.2009.06.045

2. Korostenskaja M, Kapeller C, Chen PC, et al. Estimation of Intracranial P300 Speller Sites with Magnetoencephalography (MEG)-Perspectives 
for Non-invasive Navigation of Subdural Grid Implantation. In: Guger C, Allison B, Ushiba J, eds. Brain-Computer Interface Research: A State-of-the-Art Summary 5. Springer International Publishing; 2017:111-121. http://dx.doi. org/10.1007/978-3-319-57132-4 9

3. Bamdad M, Zarshenas H, Auais MA. Application of $\mathrm{BCl}$ systems in neurorehabilitation: a scoping review. Disabil Rehabil Assist Technol. 2015;10(5):355-364. https://doi.org/10.3109/17483 $\underline{107.2014 .961569}$

4. Monge-Pereira E, Ibañez-Pereda J, Alguacil-Diego IM, Serrano JI, Spottorno-Rubio MP, Molina-Rueda F. Use of electroencephalography brain-computer interface systems as a rehabilitative approach for upper limb function after a stroke: a systematic review. PM R. 2017;9(9):918932. https://doi.org/10.1016/j.pmrj.2017.04.016

5. Bowsher K, Civillico EF, Coburn J, et al. Brain-computer interface devices for patients with paralysis and amputation: a meeting report. J Neural Eng. 2016;13(2):023001. https://doi. org/10.1088/1741-2560/13/2/023001

6. Guger C, Allison BZ, Großwindhager B, et al. How many people could use an SSVEP BCl?. Front Neurosci. 2012;6:169. https://doi. org/10.3389/fnins.2012.00169

7. Ramos-Murguialday A, Schürholz M, Caggiano V, et al. Proprioceptive feedback and brain computer interface $(\mathrm{BCl})$ based neuroprostheses. PLoS One. 2012;7(10):e47048. https://doi. org/10.1371/journal.pone.0047048

8. Sollfrank T, Ramsay A, Perdikis S, et al. The effect of multimodal and enriched feedback on SMR-BCl performance. Clin Neurophysiol. 2016;127(1):490-498. https://doi.org/10.1016/j. clinph.2015.06.004

9. Horowitz A, Guger C, Korostenskaja M. What external variables affect sensorimotor rhythm brain-computer interface (SMR-BCl) performance?. HCA Healthcare Journal of Medicine. 2021;2(3):143-162. https://doi.org/10.36518/2689$\underline{0216.1188}$

10. Friedrich EV, Scherer R, Sonnleitner K, Neuper C. Impact of auditory distraction on user performance in a brain-computer interface driven by different mental tasks. Clin Neurophysiol. 2011;122(10):2003-2009. https://doi.org/10.1016/j. clinph.2011.03.019

11. Guger C, Edlinger G, Harkam W, Niedermayer I, Pfurtscheller G. How many people are able to operate an EEG-based brain-computer interface (BCI)?. IEEE Trans Neural Syst Rehabil Eng. 2003;11(2):145-147. https://doi.org/10.1109/tnsre.2003.814481

12. Nijboer F, Birbaumer N, Kübler A. The influence of psychological state and motivation on brain-computer interface performance in patients with amyotrophic lateral sclerosis - a longitudinal study. Front Neurosci. 2010;4:55. https://doi.org/10.3389/fnins.2010.00055
13. Bian Y, Qi H, Zhao L, Ming D, Guo T, Fu X. Improvements in event-related desynchronization and classification performance of motor imagery using instructive dynamic guidance and complex tasks. Comput Biol Med. 2018;96:266-273. https://doi.org/10.1016/j.compbiomed.2018.03.018

14. Halder S, Agorastos D, Veit R, et al. Neural mechanisms of brain-computer interface control. Neuroimage. 2011;55(4):1779-1790. https:// doi.org/10.1016/j.neuroimage.2011.01.021

15. Hwang HJ, Kwon K, Im CH. Neurofeedback-based motor imagery training for brain-computer interface (BCl). J Neurosci Methods. 2009;179(1):150-156. https://doi. org/10.1016/j.jneumeth.2009.01.015

16. Mashat MEM, Lin CT, Zhang D. Effects of task complexity on motor imagery-based brain-computer interface. IEEE Trans Neural Syst Rehabil Eng. 2019;27(10):2178-2185. https://doi. org/10.1109/tnsre.2019.2936987

17. Scherer R, Faller J, Friedrich EV, et al. Individually adapted imagery improves brain-computer interface performance in end-users with disability. PLoS One. 2015;10(5):e0123727. https://doi. org/10.1371/journal.pone.0123727

18. Botrel L, Kübler A. Week-long visuomotor coordination and relaxation trainings do not increase sensorimotor rhythms (SMR) based brain-computer interface performance. Behav Brain Res. 2019;372:111993. https://doi.org/10.1016/j. bbr.2019.111993

19. Cho W, Sabathiel N, Ortner R, et al. Paired associative stimulation using brain-computer interfaces for stroke rehabilitation: a pilot study. Eur J Trans/ Myol. 2016;26(3):6132. Published 2016 Jun 6. https://doi.org/10.4081/ejtm.2016.6132

20. Emami Z, Chau T. Investigating the effects of visual distractors on the performance of a motor imagery brain-computer interface. Clin Neurophysiol. 2018;129(6):1268-1275. https://doi. org/10.1016/j.clinph.2018.03.015

21. Geronimo A, Simmons Z, Schiff SJ. Performance predictors of brain-computer interfaces in patients with amyotrophic lateral sclerosis. J Neural Eng. 2016;13(2):026002. https://doi. org/10.1088/1741-2560/13/2/026002

22. Guger C, Kapeller C, Ortner R, Kamada K. Motor Imagery with Brain-Computer Interface Neurotechnology. In: Garcia BM, ed. Motor Imagery: Emerging Practices, Role in Physical Therapy and Clinical Implications. Nova Science; 2015:61-79.

23. Guger C, Ramoser H, Pfurtscheller G. Real-time EEG analysis with subject-specific spatial patterns for a brain-computer interface (BCl). IEEE Trans Rehabil Eng. 2000;8(4):447-456. https:// doi.org/10.1109/86.895947

24. Hammer EM, Halder S, Blankertz B, et al. Psychological predictors of SMR-BCI performance. Biol Psychol. 2012;89(1):80-86. https://doi. org/10.1016/j.biopsycho.2011.09.006 
25. Hammer EM, Kaufmann T, Kleih SC, Blankertz B, Kübler A. Visuo-motor coordination ability predicts performance with brain-computer interfaces controlled by modulation of sensorimotor rhythms (SMR). Front Hum Neurosci. 2014;8:574. https://doi.org/10.3389/fnhum.2014.00574

26. Jeunet C, N'Kaoua B, Lotte F. Advances in user-training for mental-imagery-based $\mathrm{BCl}$ control: Psychological and cognitive factors and their neural correlates. Prog Brain Res. 2016;228:3-35. https://doi.org/10.1016/ bs.pbr.2016.04.002

27. Kleih SC, Kübler A. Empathy, motivation, and P300 BCl performance. Front Hum Neurosci. 2013;7:642. https://doi.org/10.3389/fnhum.2013.00642

28. Kleih S, Riccio A, Mattia D, et al. Motivation Influences Performance in SMR-BCI. In: Proc. of the 5th International Brain Computer Interface Conference 2011. Verlag der Technischen Universität Graz; 2011:108-111.

29. Leeb R, Lee F, Keinrath C, Scherer R, Bischof H, Pfurtscheller $\mathrm{G}$. Brain-computer communication: motivation, aim, and impact of exploring a virtual apartment. IEEE Trans Neural Syst Rehabil Eng. 2007;15(4):473-482. https://doi.org/10.1109/ tnsre.2007.906956

30. Meng J, Streitz T, Gulachek N, Suma D, He B. Three-dimensional brain-computer interface control through simultaneous overt spatial attentional and motor imagery tasks. IEEE Trans Biomed Eng. 2018;65(11):2417-2427. https://doi. org/10.1109/tbme.2018.2872855

31. Atassi N, Cook A, Pineda CM, Yerramilli-Rao P, Pulley D, Cudkowicz M. Depression in amyotrophic lateral sclerosis. Amyotroph Lateral Scler. 2011;12(2):109-112. https://doi.org/10.3109/1748296 8.2010 .536839

32. Dryden DM, Saunders LD, Rowe BH, et al. Depression following traumatic spinal cord injury. Neuroepidemiology. 2005;25(2):55-61. https://doi. org/10.1159/000086284

33. Jeunet C, N'Kaoua B, Subramanian S, Hachet M, Lotte F. Predicting mental imagery-based $\mathrm{BCl}$ performance from personality, cognitive profile and neurophysiological patterns. PLoS One. 2015;10(12):e0143962. https://doi.org/10.1371/journal.pone.0143962

34. Patten SB, Beck CA, Williams JV, Barbui C, Metz LM. Major depression in multiple sclerosis: a population-based perspective. Neurology. 2003;61(11):1524-1527. https://doi.org/10.1212/01. wnl.0000095964.34294.b4

35. Thomschewski A, Ströhlein A, Langthaler PB, et al. Imagine There is no plegia. Mental motor imagery difficulties in patients with traumatic spinal cord injury. Front Neurosci. 2017;11:689. https://doi.org/10.3389/fnins.2017.00689

36. Ahn M, Cho H, Ahn S, Jun SC. High theta and low alpha powers may be indicative of
$\mathrm{BCl}$-illiteracy in motor imagery. PLoS One. 2013;8(11):e80886. https://doi.org/10.1371/journal. pone.0080886

37. Ang KK, Guan C. EEG-based strategies to detect motor imagery for control and rehabilitation. IEEE Trans Neural Syst Rehabil Eng. 2017;25(4):392-401. https://doi.org/10.1109/tnsre.2016.2646763

38. Azab AM, Mihaylova L, Ang KK, Arvaneh M. Weighted transfer learning for improving motor imagery-based brain-computer interface. IEEE Trans Neural Syst Rehabil Eng. 2019;27(7):13521359. https://doi.org/10.1109/tnsre.2019.2923315

39. Bamdadian A, Guan C, Ang KK, Xu J. The predictive role of pre-cue EEG rhythms on $\mathrm{Ml}$-based $\mathrm{BCl}$ classification performance. J Neurosci Methods. 2014;235:138-144. https://doi.org/10.1016/j. jneumeth.2014.06.011

40. Belwafi K, Gannouni S, Aboalsamh H, Mathkour $\mathrm{H}$, Belghith A. A dynamic and self-adaptive classification algorithm for motor imagery EEG signals. J Neurosci Methods. 2019;327:108346. https://doi.org/10.1016/j.jneumeth.2019.108346

41. Blankertz B, Sannelli C, Halder S, et al. Neurophysiological predictor of SMR-based $\mathrm{BCl}$ performance. Neuroimage. 2010;51(4):1303-1309. https://doi.org/10.1016/j.neuroimage.2010.03.022

42. Dinarès-Ferran J, Ortner R, Guger C, Solé-CasaIs J. A new method to generate artificial frames using the empirical mode decomposition for an EEG-based motor imagery $\mathrm{BCl}$. Front Neurosci. 2018;12:308. https://doi.org/10.3389/ fnins.2018.00308

43. Gaur P, McCreadie K, Pachori RB, Wang H, Prasad G. Tangent space features-based transfer learning classification model for two-class motor imagery brain-computer interface. Int J Neural Syst. 2019;29(10):1950025. https://doi. org/10.1142/s0129065719500254

44. Grosse-Wentrup M, Schölkopf B. High y-power predicts performance in sensorimotor-rhythm brain-computer interfaces. J Neural Eng. 2012;9(4):046001. https://doi.org/10.1088/17412560/9/4/046001

45. Guan S, Zhao K, Yang S. Motor Imagery EEG Classification based on decision tree framework and Riemannian geometry. Comput Intell Neurosci. 2019;2019:5627156. https://doi. org/10.1155/2019/5627156

46. Joadder MAM, Myszewski JJ, Rahman MH, Wang I. A performance based feature selection technique for subject independent MI based BCl. Health Inf Sci Syst. 2019;7(1):15. https://doi. org/10.1007/s13755-019-0076-2

47. Olias J, Martin-Clemente R, Sarmiento-Vega MA, Cruces S. EEG Signal processing in MI-BCl applications with improved covariance matrix estimators. IEEE Trans Neural Syst Rehabil Eng. 2019;27(5):895-904. https://doi.org/10.1109/tnsre.2019.2905894 
48. Robinson N, Thomas KP, Vinod AP. Neurophysiological predictors and spectro-spatial discriminative features for enhancing SMR-BCI. J Neural Eng. 2018;15(6):066032. https://doi. org/10.1088/1741-2552/aae597

49. Vidaurre C, Sannelli C, Müller KR, Blankertz B. Co-adaptive calibration to improve $\mathrm{BCl}$ efficiency. J Neural Eng. 2011;8(2):025009. https://doi. org/10.1088/1741-2560/8/2/025009

50. Zhang L, Wei Q. Channel selection in motor imaginary-based brain-computer interfaces: a particle swarm optimization algorithm. $J$ Integr Neurosci. 2019;18(2):141-152. https://doi. org/10.31083/j.jin.2019.02.17

51. Zhang R, Xu P, Chen R, et al. Predicting inter-session performance of SMR-based brain-computer interface using the spectral entropy of resting-state EEG. Brain Topogr. 2015;28(5):680-690. https://doi.org/10.1007/ s10548-015-0429-3

52. Zhang T, Liu T, Li F, et al. Structural and functional correlates of motor imagery $\mathrm{BCl}$ performance: Insights from the patterns of fronto-parietal attention network. Neuroimage. 2016;134:475-485. https://doi.org/10.1016/j. neuroimage.2016.04.030

53. Zhang Y, Nam CS, Zhou G, Jin J, Wang X, Cichocki A. Temporally constrained sparse group spatial patterns for motor imagery $\mathrm{BCl}$. IEEE Trans Cybern. 2019;49(9):3322-3332. https://doi. org/10.1109/tcyb.2018.2841847

54. Blankertz B, Losch F, Krauledat M, Dornhege G, Curio G, Müller KR. The Berlin Brain--Computer Interface: accurate performance from first-session in $\mathrm{BCl}$-naïve subjects. IEEE Trans Biomed Eng. 2008;55(10):2452-2462. https://doi. org/10.1109/tbme.2008.923152

55. Silva S, Borges LR, Santiago L, Lucena L, Lindquist AR, Ribeiro T. Motor imagery for gait rehabilitation after stroke. Cochrane Database Syst Rev. 2020;9:CD013019. https://doi. org/10.1002/14651858.cd013019.pub2

56. Talukdar U, Hazarika SM, Gan JQ. Motor imagery and mental fatigue: inter-relationship and EEG based estimation. J Comput Neurosci. 2019;46(1):55-76. https://doi.org/10.1007/s10827018-0701-0

57. Kraeutner SN, Keeler LT, Boe SG. Motor imagery-based skill acquisition disrupted following rTMS of the inferior parietal lobule. Exp Brain Res. 2016;234(2):397-407. https://doi.org/10.1007/ s00221-015-4472-9

58. Ahn M, Cho H, Ahn S, Jun SC. User's self-prediction of performance in motor imagery brain-computer interface. Front Hum Neurosci. 2018;12:59. https://doi.org/10.3389/fnhum.2018.00059

59. Rimbert S, Gayraud N, Bougrain L, Clerc M, Fleck S. Can a subjective questionnaire be used as brain-computer interface performance pre- dictor?. Front Hum Neurosci. 2019;12:529. https:// doi.org/10.3389/fnhum.2018.00529

60. Darvishi S, Gharabaghi A, Ridding MC, Abbott $D$, Baumert $M$. Reaction time predicts brain-computer interface aptitude. IEEE J Trans/ Eng Health Med. 2018;6:2000311. https://doi. org/10.1109/jtehm.2018.2875985

61. Meng J, Mundahl J, Streitz T, et al. Effects of soft drinks on resting state EEG and brain-computer interface performance. IEEE Access. 2017;5:18756-18764. https://doi.org/10.1109/access.2017.2751069

62. Zhang R, Yao D, Valdés-Sosa PA, et al. Efficient resting-state EEG network facilitates motor imagery performance. J Neural Eng. 2015;12(6):066024. https://doi.org/10.1088/17412560/12/6/066024

63. Jeunet C, Jahanpour E, Lotte F. Why standard brain-computer interface $(\mathrm{BCl})$ training protocols should be changed: an experimental study. J Neural Eng. 2016;13(3):036024. https://doi. org/10.1088/1741-2560/13/3/036024

64. Cantillo-Negrete J, Gutierrez-Martinez J, Carino-Escobar RI, Carrillo-Mora P, Elias-Vinas D. An approach to improve the performance of subject-independent $\mathrm{BCl}$-based on motor imagery allocating subjects by gender. Biomed Eng Online. 2014;13:158. Published 2014 Dec 4. https:// doi.org/10.1186/1475-925x-13-158

65. Skrandies W, Klein A. Brain activity and learning of mathematical rules--effects on the frequencies of EEG. Brain Res. 2015;1603:133-140. https:// doi.org/10.1016/j.brainres.2014.11.015 